\title{
Tres elementos fundamentales 1 en la formación de docentes de ciencias
}

\section{- Three Fundamental Elements in the Training of Science Teachers}

- Três elementos fundamentais na formação de professores de ciências

\section{Vicente Talanquer ${ }^{*}$}

\section{Resumen}

En este ensayo se describen, analizan y discuten tres elementos formativos que se consideran fundamentales en la preparación docente, primero la conceptualización de la disciplina como forma productiva de pensar y actuar sobre el mundo; segundo, la habilidad para seleccionar y/o diseñar tareas instruccionales retadoras y activas que promuevan la construcción de explicaciones y/o soluciones a fenómenos o problemas concretos de interés. Finalmente la capacidad de implementar tareas instruccionales de manera responsiva, desde un modelo de evaluación formativa, para apoyar, enriquecer, cuestionar y dirigir el pensamiento.

Se analiza que un proceso de enseñanza centrado en estos aspectos satisface múltiples metas de la educación en ciencias, tales como, desarrollar comprensiones significativas de conceptos e ideas centrales: promover la participación en diversas prácticas científicas; involucrar la argumentación y otras formas discursivas empleadas en la construcción y evaluación del conocimiento científico; diseñar soluciones de interés para estudiantes y la sociedad. Además de crear oportunidades de aprendizaje con un nivel apropiado de reto intelectual.

Finalmente se reflexiona sobre la enseñanza responsiva de la disciplina, como medio para facilitar la participación equitativa y favorecer el desarrollo profesional de los docentes, quienes aprenden a responder de manera productiva a las ideas de sus estudiantes en situaciones específicas y contextos variados.

Palabras clave

Formación docente; planeación; instrucción; evaluación formativa

\begin{abstract}
This paper describes, analyzes and discusses three educational elements that are considered fundamental in teacher training: first, the conceptualization of the discipline as a productive way of thinking and acting about the world; second, the ability to select and/or design challenging and active instructional tasks encouraging the development of explanations and/or solutions to specific phenomena or problems of interest; finally, the ability to implement instructional
\end{abstract}

Profesor Distinguido del Departamento de Química y Bioquímica de la Universidad de Arizona en los Estados Unidos. Correo electrónico: vicente@u.arizona.edu, Orcid: 0000-0002. 5737-3313 
tasks in a responsive manner from an educational evaluation model so as to support, enrich, question and guide the way of thinking.

The article analyzes the fact that a teaching process focused in these aspects fulfills multiple science education goals, such as developing meaningful understanding of central concepts and ideas; encouraging the participation in several scientific practices; involving the argumentation and other discursive forms used in the development and evaluation of scientific knowledge; designing solutions of interest for students and society; and creating learning opportunities with an appropriate level of intellectual challenge.

Finally, a reflection is made on the responsive teaching of the discipline as a means to facilitate equitable participation and favor the professional development of teachers, who learn to productively respond to the ideas of their students in specific situations and varied contexts.

Keywords

Teacher training; planning; instruction; formative evaluation

\section{Resumo}

Neste ensaio são descritos, analisados e discutidos três elementos formativos que se consideram fundamentais na preparação docente: primeiro, a conceptualização da disciplina como forma produtiva de pensar e atuar sobre o mundo; segundo, a habilidade para selecionar ou planejar tarefas instrucionais desafiantes e ativas que possam promover a construção de explicações ou soluções para fenômenos ou problemas concretos de interesse; finalmente, a capacidade de implementar tarefas instrucionais de forma responsiva, desde um modelo de avaliação formativa para apoiar, enriquecer, questionar e dirigir o pensamento.

Observa-se que um processo de ensino centrado nestes aspectos satisfaz diversos objetivos da educação em ciências como: desenvolver compreensões significativas de conceptos e ideias centrais; promover a participação em diversas práticas científicas; envolver a argumentação e outras formas discursivas empregadas na construção e avaliação do conhecimento cientifico; planejar soluções de interesse para estudantes e a sociedade, além de criar oportunidades de aprendizagem com um nível apropriado de reto intelectual.

Finalmente, reflete-se sobre o ensino responsivo da disciplina como meio para facilitar a participação equitativa e favorecer o desenvolvimento profissional dos professores, que aprendem a responder de forma produtiva às ideias dos seus estudantes em situações específicas e contextos diversos.

Palavras chave

Formação docente; planejamento; instrução; avaliação formativa 


\section{Introducción}

A pesar de las múltiples reformas educativas en la enseñanza de las ciencias promovidas e implementadas en diferentes partes del mundo en los últimos cuarenta años, la actividad docente en la mayoría de las aulas en diversos niveles educativos sigue estando dominada por la transmisión pasiva de información (OECD, 2009). Esta tradicionalmente se organiza de acuerdo a lo que los expertos en cada área consideran como la secuenciación lógica del conocimiento en su disciplina. Muchos docentes tienden a conceptualizar al currículo como un listado de temas por cubrir en un tiempo determinado, acompañado de un conjunto de actividades complementarias (resolver problemas, hacer prácticas de laboratorio, presentar demostraciones de cátedra) (Porlan y Del Pozo, 2004). En general, los estándares y prácticas de enseñanza que emanan de las distintas reformas educativas acaban siendo adoptados de manera cosmética a través de cambios menores en la organización del temario, los ejemplos que los docentes utilizan para ilustrar algunos conceptos, o las prácticas de laboratorio que se implementan (Roehrig y Kruse, 2005).

La dificultad o resistencia para implementar auténticos cambios en el aula emerge de la combinación de múltiples y variados factores de tipo individual, escolar, social, económico y político. Los docentes, por ejemplo, tienen creencias particulares sobre los propósitos de la enseñanza, las mejores formas de aprender - las estrategias de enseñanza más efectivas (Fang, 1996). Estos docentes trabajan en ambientes escolares particulares y perciben, experimentan y responden de manera distinta a las reformas educativas que se les plantean o imponen periódicamente (Darling-Hammond y Brandsford, 2005). Muchos de ellos no reciben los apoyos y la preparación suficientes y necesarios para implementar los cambios promovidos. Todo esto hace que el futuro de las reformas educativas descanse en gran medida en las fortalezas y debilidades en la formación inicial de los docentes encargados de implementarlas.

Dado el rol central que la preparación del profesorado desempeña en el futuro de las reformas educativas, en este artículo centro mi atención en lo que considero como aspectos críticos en la formación de docentes de ciencias. Mis ideas se basan tanto en la literatura existente sobre el tema como en mis propias experiencias como formador de maestros de ciencias para los niveles medio, medio superior y superior en la Universidad de Arizona. Es probable que mis propuestas les parezcan limitadas o insuficientes a muchos lectores porque se centran en un conjunto reducido de elementos formativos. Sin embargo, mi intención es resaltar aquellos aspectos que, desde mi perspectiva, son necesarios y potencialmente más productivos en la formación de docentes con la capacidad de implementar el tipo de cambios que sabemos son más efectivos en la promoción de aprendizajes significativos en salones de clases caracterizados por la diversidad de los intereses, motivaciones, acceso a recursos y capital cultural de su población estudiantil.

\section{Áreas críticas en la formación docente}

Las propuestas de reforma en educación de las ciencias desarrolladas en diversos países en años recientes comparten un gran número de objetivos y estrategias (NRC, 2011, 2013; Osborne y Dillon, 2008). Se enfatiza, por ejemplo, la importancia de crear oportunidades para que los estudiantes se involucren de manera activa en la aplicación, construcción y evaluación de modelos para generar explicaciones sobre fenómenos interesantes 
y relevantes para los individuos y las sociedades en las que viven. Se resalta la necesidad de integrar el desarrollo de conocimientos científicos con el de distintas prácticas científicas, como hacer preguntas, planear investigaciones y plantear y defender argumentos para resolver problemas y construir explicaciones. Los estándares educativos se definen no en términos de contenidos a cubrir, sino en función de competencias o expectativas de desempeño que describen el tipo de formas de pensar y actuar que se espera que los estudiantes demuestren al finalizar sus estudios.

En este contexto, los docentes se enfrentan con el reto de crear oportunidades de aprendizaje que ayuden a los estudiantes a construir ideas centrales y a desarrollar prácticas científicas de manera integrada. Su éxito, desde mi perspectiva, depende de la habilidad del docente para:

- Identificar las preguntas esenciales y las ideas, quehaceres y formas de pensar centrales en su disciplina.

- Diseñar e implementar tareas instruccionales que involucren a los estudiantes de manera activa y sostenida a un alto nivel en la construcción y evaluación de tales ideas y formas de pensar y actuar.

- Implementar evaluación formativa de manera continua y responsiva.

En las siguientes secciones elaboro cada uno de estos elementos formativos.

\section{Conceptualización de la disciplina}

La gran mayoría de las personas que terminan una carrera de ciencias, o sobre la enseñanza de una disciplina científica, conciben su materia como el conjunto de conocimientos al que han sido expuestos durante su formación profesional (Gess-Newsome, 1999; Mora y Parga, 2008). Para ellos, aprender sobre la disciplina con frecuencia significa aprender sobre los temas en los que sus estudios profesionales tradicionalmente se dividen. Aprender física, por ejemplo, se trata de saber sobre mecánica, óptica y electromagnetismo. En biología hay que aprender sobre evolución, genética y ecología. En química hay que estudiar estructura de la materia, termodinámica y cinética. No es de extrañar entonces que, al enfrentarse con la tarea de enseñar a otros, los cursos que estos profesionistas diseñan e implementan tengan como objetivo la transmisión de dichos contenidos disciplinarios.

Esta visión de las disciplinas científicas no representa de manera adecuada sus propósitos y alcances, ni lo que significa trabajar y pensar con y dentro de esas áreas. Una disciplina se define menos por los contenidos que abarca que por los tipos de preguntas que nos permite responder, los problemas que nos ayuda a resolver y las estrategias intelectuales y experimentales que se utilizan para recabar información, organizarla, analizarla y aplicarla. La diferencia entre un novato y un experto en una cierta área tiene menos que ver con la cantidad 
de información que cada uno ha memorizado que con su habilidad para utilizarla de manera productiva en variados contextos (Roberson y Franchini, 2014).

Un docente que conceptualiza su disciplina como una forma particular de pensar y conocer el mundo se encuentra en una mejor posición para diseñar oportunidades de aprendizaje significativas que uno que la concibe como un conjunto estático de conocimientos. El primero estará más abierto y dispuesto al desarrollo de tareas instruccionales que enfrenten a los estudiantes con la resolución de problemas más auténticos. Este docente prestará más atención a las formas de pensar y actuar de sus estudiantes. Sus evaluaciones seguramente incluirán un mayor número de instancias en las que estos deban tomar decisiones, evaluar alternativas, proponer soluciones y generar explicaciones.

Pocos programas de formación de docentes involucran a sus estudiantes en la reflexión crítica sobre la naturaleza de su disciplina, sus propósitos, las acciones que la definen y su impacto en el mundo que nos rodea (Vilches y Gil, 2007). Si se dan, las reflexiones de carácter histórico, filosófico, sociológico y político típicamente se constriñen a aspectos educativos desligados de la naturaleza del contenido a enseñar. Sin embargo, es de central importancia que los docentes se cuestionen:

- $\quad \dot{Q}$ ué tipos de problemas nos ayuda a resolver la disciplina?

- $\quad$ ¿Cómo se obtiene y usa la información que nos permite resolver tales problemas?

- $\quad \dot{Q}$ ué tipos de juicios y decisiones se toman para resolver problemas en la disciplina?

- $\quad$ ¿Qué tipos de suposiciones se hacen?
- ¿ ¿Qué ideas centrales guían la toma de decisiones y la generación de explicaciones?

- $\quad$ ¿Qué tipos de herramientas intelectuales y experimentales se utilizan?

- ¿ ¿Cuáles son los beneficios, costos y riesgos asociados con el trabajo y los productos de la disciplina?

La búsqueda individual y colectiva de respuestas a estas preguntas y la discusión de sus implicaciones para la enseñanza de la materia es necesaria si queremos cuestionar y transformar las visiones tradicionales que dominan la práctica docente.

Mi experiencia personal como formador de docentes de química para los diferentes niveles educativos me deja claro que el tipo de reflexión que sugiero no es fácil. Las limitaciones en el conocimiento y las experiencias de los maestros con los que normalmente trabajo dificultan la reflexión crítica. Es por ello que he optado por involucrar a los docentes en formación en el trabajo en un curso introductorio de química a nivel universitario en el que la organización curricular y las tareas instruccionales modelan una forma diferente de conceptualizar la disciplina (Talanquer, 2013). Las reflexiones y discusiones se facilitan a través del análisis de experiencias y ejemplos concretos en el salón de clases.

El curso de referencia, denominado Pensamiento Químico, ha sido diseñado buscando introducir la química como una forma productiva de pensar para responder preguntas y resolver problemas de importancia en el mundo que nos rodea (Talanquer y Pollard, 2010). De manera no convencional, el currículo está organizado alrededor de ocho preguntas esenciales (véase la tabla 1) y el trabajo en el aula se centra en la identificación, construcción, discusión y evaluación de ideas y estrategias 
(intelectuales y experimentales) que permiten darles respuesta en diversos contextos. Por ejemplo, en la Unidad 1 las discusiones se dirigen al análisis de conceptos y métodos que nos permiten diferenciar y separar sustancias en sistemas de interés, como la atmósfera de nuestro planeta, el agua que bebemos o la sangre en nuestro cuerpo.

Tabla 1. Preguntas esenciales que dirigen el trabajo en el curso Pensamiento Químico

\begin{tabular}{|c|c|}
\hline Unidad & Pregunta esencial \\
\hline 1 & ¿Cómo diferenciamos sustancias? \\
\hline 2 & ¿Cómo determinamos estructura? \\
\hline 3 & ¿̇Cómo predecimos propiedades? \\
\hline 4 & ¿̇Cómo caracterizamos reacciones químicas? \\
\hline 5 & ¿̇Cómo predecimos procesos químicos? \\
\hline 6 & ¿̇Cómo controlamos procesos químicos? \\
\hline 7 & ¿Cómo aprovechamos la energía química? \\
\hline 8 & ¿̇Cómo sintetizamos nuevas sustancias? \\
\hline
\end{tabular}

Fuente: elaboración propia

La estructura, los objetivos de aprendizaje, las tareas instruccionales y las evaluaciones que se utilizan en el curso Pensamiento Químico buscan resaltar las formas de razonar que facilitan la construcción de explicaciones y predicciones sobre las propiedades y el comportamiento de las sustancias químicas, y que nos ayudan a analizarlas, transformarlas y sintetizarlas. El aprendizaje del contenido no se plantea como un fin en sí mismo, sino como un sustento para las formas de pensar que queremos desarrollar. Este cambio de enfoque crea oportunidades para reflexionar sobre lo que se enseña y para qué se enseña. Dada una pregunta esencial para contestar, el docente debe considerar qué conceptos, ideas y habilidades son necesarios para enfrentar el problema. Debe identificar contextos relevantes para sus estudiantes en los que hacerse tal pregunta es de interés central. Y debe seleccionar o diseñar tareas instruccionales que ayuden a sus estudiantes a desarrollar las formas de pensar que se valoran, no solo los conocimientos que se requieren. Como se discute en la siguiente sección, la habilidad de los docentes para seleccionar o diseñar este tipo de tareas instruccionales es el segundo elemento formativo que sugiero considerar.

\section{Las tareas instruccionales}

El término "tarea instruccional" en educación típicamente se refiere a unidades de trabajo que organizan y dirigen el pensamiento y las acciones de los estudiantes en el aula (Doyle y Carter, 1984). Estas tareas organizan la labor cognitiva a través de la definición de las metas o productos esperados, los recursos disponibles para completarlas y el conjunto de operaciones que se deben aplicar. Las oportunidades que los estudiantes tienen para aprender se incrementan cuando trabajan en tareas instruccionales que representan un reto intelectual apropiado para su nivel, y se implementan asegurando que exista alta demanda cognitiva 
de forma sostenida. Las tareas instruccionales influencian el aprendizaje a través de su efecto en cómo los estudiantes piensan en los problemas o preguntas planteados, el tipo de conversaciones que se establecen en el aula, las actividades en las que estos se involucran y las ideas, creencias y respuestas emotivas que desarrollan sobre los propósitos del aprendizaje (Thompson, 2014).

Varios investigadores en educación de las ciencias han identificado rasgos fundamentales de tareas instruccionales con alta capacidad para promover el aprendizaje significativo de conceptos, habilidades y prácticas científicas (Chi y Wylie, 2014; Kang et al., 2016; Roberson y Franchini, 2014). Sin embargo, unas cuantas visitas a salones de clase desde los niveles primarios hasta el posgrado bastan para darse cuenta de que pocos docentes involucran a sus estudiantes en tareas con tales características. Por ello es de central importancia que los docentes en formación tengan múltiples oportunidades para observar, construir, implementar, evaluar y reflexionar sobre tareas instruccionales que estén mejor alineadas con principios de diseño fundamentados en los resultados de la investigación educativa en su disciplina.

Las tareas instruccionales más efectivas típicamente enfrentan a los estudiantes con situaciones, fenómenos o problemas complejos que son de su interés y significan un reto intelectual para ellos. Trabajan en estas tareas durante varios días, desarrollando e integrando múltiples conceptos e ideas (Windschitl et al., 2012). Estas tareas buscan crear un nivel adecuado de incertidumbre cognitiva, epistémica y procedimental para los estudiantes. Esta incertidumbre en qué y cómo pensar y actuar para enfrentar la situación planteada abre oportunidades para que los estudiantes se planteen preguntas, generen hipótesis, construyan modelos y argumenten ideas
(García y Rentería, 2011 ; Kang et al., 2016). Estas tareas se plantean como una búsqueda de explicaciones o soluciones a fenómenos o problemas específicos en contextos concretos y no como actividades genéricas sin más propósito que el ejercicio intelectual o manual que proporcionan.

Los docentes deben aprender a diseñar y presentar estas tareas instruccionales de manera que los estudiantes se conecten con ellas y puedan reconocer la relación entre el fenómeno por estudiar o el problema por resolver y los conceptos, ideas y prácticas científicas que será necesario aprender para enfrentar el reto. En la medida de lo posible, estas tareas deben ser seleccionadas o diseñadas prestando atención a las experiencias e intereses de los estudiantes con los que se trabaja y no simplemente porque se recomiendan en un libro de texto o en los materiales curriculares disponibles. Las tareas deben crear múltiples oportunidades para explorar, introducir, aplicar e integrar ideas y prácticas científicas en la disciplina.

Una buena tarea instruccional debe involucrar a los estudiantes de manera activa y frecuente en la toma de decisiones que hagan visible su comprensión y razonamiento sobre los conceptos y habilidades que se busca desarrollar (Roberson y Franchini, 2014). Los juicios que se demanda que los estudiantes hagan deben replicar en la medida de lo posible los tipos de razonamientos que los expertos en el área aplicarían si se enfrentaran con un problema auténtico del mismo tipo, en condiciones similares. Desde este punto de vista, tareas instruccionales que se pueden completar simplemente recordando definiciones, aplicando algoritmos, expresando opiniones o describiendo experiencias personales no deben ser las actividades dominantes en un salón de clases.

En general, los docentes deben aprender y aspirar a construir tareas instruccionales que 
involucren a los estudiantes en una variedad de prácticas científicas y demanden la integración de conceptos e ideas con el fin de construir explicaciones causales de fenómenos concretos o diseñar y justificar soluciones a problemas específicos de interés (Russ et al., 2009). Estas tareas típicamente requieren que los estudiantes colaboren en la recolección, análisis y evaluación de datos de distintos tipos y representados de distintas formas. Se busca que utilicen, co-construyan, evalúen y revisen modelos con diferentes niveles de abstracción (Chi y Wylie, 2014). El trabajo debe demandar que los estudiantes identifiquen evidencia relevante y construyan argumentos para justificar las explicaciones o soluciones propuestas. Es importante reconocer que la selección o el diseño de una buena tarea instruccional es un requisito necesario pero no suficiente para crear oportunidades de aprendizaje de alto nivel. También se requiere que el docente la implemente de manera productiva. Las características de una implementación adecuada se describen y discuten en la siguiente sección.

\section{La evaluación formativa como marco de referencia y acción}

La implementación de tareas instruccionales de forma efectiva demanda que los docentes se embarquen en evaluación formativa de manera continua y responsiva. Concibo la evaluación formativa como un proceso dinámico en el que el docente obtiene información sobre el aprendizaje en el aula, la interpreta y evalúa, y crea condiciones y proporciona herramientas para que los estudiantes avancen en su comprensión de las ideas y habilidades que al maestro le interesan (Black y Wiliam, 1998, 2009; Bennett, 2011). La evaluación formativa demanda que el docente esté atento a las ideas expresadas por los estudiantes, las interprete de manera productiva y tome acciones encaminadas a apoyar el desarrollo de aprendizajes significativos (Cowie y Bell, 1999). La evaluación formativa responsiva requiere que el docente conciba, reconozca y utilice las ideas y experiencias de los estudiantes como recursos que pueden apoyar el aprendizaje (Robertson, Scherr y Hammer, 2016).

Los resultados de la investigación educativa en diversas áreas nos permiten identificar el tipo de interacciones formativas que tienen un mayor potencial para involucrar a la mayoría de los estudiantes en trabajo intelectual productivo (Coffey et al., 2011 ). Algunos autores se refieren a estas interacciones como prácticas docentes que sustentan la enseñanza ambiciosa de la disciplina (Windschitl et al., 2012). Estas prácticas incluyen hacer visibles las ideas de los estudiantes con el fin de adaptar la enseñanza, la inclusión de andamiajes para apoyar cambios progresivos en las formas de pensar de los estudiantes y la demanda continua de explicaciones generadas con base en evidencias.

Toda tarea instruccional debe incluir actividades que activen los conocimientos y experiencias previos de los estudiantes sobre el tema de interés y hagan visibles sus formas de pensar y el lenguaje que utilizan para comunicar sus ideas (Shepard, 2005). Estas actividades por lo general involucran a los estudiantes en 
la observación y exploración de fenómenos y los invitan a generar hipótesis y modelos iniciales sobre los sistemas que estudian. Se espera que el docente cree espacios en los que los estudiantes se sientan cómodos expresando lo que piensan. El maestro debe estar dispuesto a escuchar lo que los estudiantes tienen que decir, no con el fin de evaluar si es correcto o incorrecto sino para tratar de darle sentido, interpretarlo con base en sus conocimientos sobre ideas alternativas y encontrar semillas intelectuales que sirvan como sustento para la construcción de conocimientos, habilidades o actitudes más alineadas con el saber y el quehacer disciplinarios (Levin y Richards, 2011 ; Robertson, Scherr y Hammer, 2016). La evaluación formativa se debe conceptualizar como un acto de construcción y no de corrección del pensamiento de los estudiantes (Talanquer, Bolger y Tomanek, 2015).

Durante la implementación de la tarea instruccional, los docentes deben crear variadas oportunidades para que los estudiantes razonen a través de conversaciones en las que ideas alternativas puedan ser debatidas y retroalimentación formativa pueda ser proporcionada. Estas conversaciones son también necesarias para introducir nuevos conceptos que motiven la revisión de hipótesis y modelos iniciales. La introducción de conceptos se facilita y es más efectiva cuando los estudiantes tienen la oportunidad de interactuar con datos o nueva información que requiere explicación (Windschitl et al., 2012). Estos datos pueden ser generados de manera experimental por los estudiantes, recolectados por alguien más o producidos haciendo uso de simuladores computacionales. La mayoría de los estudiantes tienen la capacidad de analizar resultados e identificar patrones de comportamiento, pero se les dificulta construir nuevos conceptos con base en ello. El docente desempeña un papel central en la introducción de conceptos abstractos que ayuden a los estudiantes a darle sentido a lo que observan. Estos productos intelectuales de la actividad en el aula constituyen la base sobre la que los estudiantes deben evaluar sus modelos y explicaciones iniciales y construir argumentos para justificar la necesidad de modificarlos.

El docente debe aprender a escuchar a los estudiantes para provocar su razonamiento de manera continua, involucrándolos en el proceso de construir argumentos con base en la evidencia disponible. Se espera que el maestro cree una cultura en el aula en la que los estudiantes entienden la importancia de utilizar múltiples fuentes de información para construir explicaciones o proponer soluciones. Este trabajo frecuentemente requiere el uso de andamiajes conceptuales y procedimentales que en un inicio guíen el trabajo y las formas de pensar de los estudiantes, pero se puedan retirar una vez que aquellos han desarrollado bases de conocimientos y habilidades más sólidas (Shepard, 2005). Se espera que estos andamiajes formativos no solo apoyen sino también problematicen el contenido disciplinario, haciendo visibles la profundidad y complejidad que los estudiantes deben desarrollar y demostrar en su trabajo.

En general, la implementación efectiva de evaluación formativa durante las tareas instruccionales requiere que el docente conozca y utilice de la mejor manera una variedad de herramientas discursivas para provocar, sostener y dirigir conversaciones en el aula centradas en el análisis, la discusión y reflexión de conceptos e ideas científicas centrales. Estas herramientas incluyen, entre otras, preguntas de sondeo que hagan visibles y públicas las ideas de los estudiantes, preguntas que soliciten la generación de explicaciones, parafraseo de ideas expresadas por los estudiantes para aclarar significados y resaltar puntos de importancia, invitaciones a establecer conversaciones entre 
pares, confirmaciones o reorientaciones que dirijan el pensamiento de los estudiantes, ligas cognitivas que hagan visibles conexiones entre conceptos e ideas y modelaciones que ilustren las formas de razonar que se valoran (Mercer, 2000; Van de Pol, Volman, Beishuizen, 2010).

\section{Comentarios finales}

La inversión en formación docente es crítica para promover y facilitar la implementación fidedigna de reformas educativas que se sustentan en resultados de la investigación educativa sobre cómo promover aprendizajes significativos y transferibles en la enseñanza de las ciencias. En este ensayo he buscado resaltar tres elementos formativos que, desde mi perspectiva, son fundamentales para que los docentes puedan crear oportunidades de aprendizaje de alta calidad en cualquier nivel educativo. Estos elementos incluyen: (1) la conceptualización de la disciplina no como un cuerpo de conocimientos especializados sino como una forma productiva de pensar y actuar sobre el mundo que nos rodea, (2) la habilidad para seleccionar o diseñar tareas instruccionales retadoras que involucren a los estudiantes de manera activa en la construcción de explicaciones y soluciones a fenómenos o problemas concretos de interés, y (3) la capacidad para implementar dichas tareas instruccionales de manera responsiva, usando evaluación formativa de manera continua para hacer visible, promover, apoyar, enriquecer, cuestionar y dirigir el pensamiento de los estudiantes.

Estos elementos y prácticas son necesarios para crear e implementar tareas instruccionales con un nivel apropiado de reto intelectual para los estudiantes, el cual pueda ser sostenido a lo largo de toda unidad de trabajo. La enseñanza centrada en estos elementos satisface múltiples metas de la educación de las ciencias en la actualidad, tales como desarrollar la comprensión significativa de conceptos e ideas centrales en cada disciplina, promover la participación de los estudiantes en diversas prácticas científicas, involucrarlos en argumentación y otras formas discursivas utilizadas en la construcción y evaluación del conocimiento científico, e integrar conocimientos y prácticas científicas en la generación de explicaciones y el diseño de soluciones de interés para los estudiantes y las sociedades en las que viven. La enseñanza ambiciosa y responsiva de la disciplina también facilita la participación equitativa de todo tipo de estudiantes en el aula y favorece el desarrollo profesional continuo de los docentes involucrados, quienes deben aprender a responder de manera productiva a las ideas de sus estudiantes en situaciones específicas y contextos variados.

La transformación que esperamos en la enseñanza de las ciencias demanda que los docentes modifiquen su forma de pensar sobre la disciplina, el tipo de tareas en las que involucran a los estudiantes, su rol en la implementación del trabajo en el aula y la evaluación del aprendizaje. El reto para la formación del profesorado parece formidable y sin duda demanda considerable inversión de tiempo y recursos. Sin embargo, en la actualidad existen modelos de formación 
docentes fundamentados en estas ideas que pueden facilitar el trabajo y que han generado recursos diversos para apoyar el trabajo de los maestros y sus formadores (TAST, 2016).

La implementación de las ideas avanzadas en este artículo también demanda que los formadores de docentes transformen su manera de pensar sobre los tipos de conocimientos que los maestros en formación y en servicio deben desarrollar. En general, nosotros también tendemos a concebir el conocimiento didáctico y pedagógico en nuestras disciplinas como un conjunto de información, conceptos, teorías, principios y prácticas establecidas que los docentes deben conocer. Rara vez representamos la didáctica disciplinaria como una forma de pensar y actuar que nos permite, entre otras cosas, crear oportunidades para aprender, generar interpretaciones sobre eventos educativos y construir soluciones a problemas de enseñanza y aprendizaje.

Los formadores de maestros debemos generar tareas instruccionales en las que los docentes en formación se enfrenten con situaciones y problemas similares a los que encontrarán en el salón de clases. Es a través del análisis, la discusión y la reflexión de eventos educativos auténticos que los maestros deben no sólo adquirir los conocimientos deseados sino hacer uso de ellos para aplicar, construir y evaluar modelos de instrucción y aprendizaje que los ayuden a atender mejor las ideas de sus estudiantes, explicar diversas situaciones didácticas, interpretar el pensamiento y el actuar de sus estudiantes y justificar y realizar acciones para promover el progreso de estos con base en la evidencia disponible. Durante este proceso, es de central importancia que evaluemos formativamente el trabajo de los docentes proporcionando la información, retroalimentación y los andamiajes necesarios para sostener, estructurar y enriquecer su trabajo

El tipo de docente que requerimos debe tener la capacidad de utilizar sus conocimientos de manera flexible y dinámica. Su tarea en el salón de clases demandará no solo que conozca algunos métodos de instrucción o esté consciente de las ideas alternativas que los estudiantes más comúnmente expresan en un tema dado. También requerirá que reconozca formas productivas de pensar en ellos, que tenga la capacidad de identificar posibles conexiones entre las ideas y experiencias cotidianas de los estudiantes y las ideas y prácticas científicas que se busca desarrollar, que identifique trayectorias de instrucción que mejor apoyen el progreso de sus estudiantes y que evalúe sus ideas buscando darles sentido y reconociendo su valor y posible contribución a la construcción de conocimientos disciplinarios.

Los docentes deben prepararse para reconocer que la enseñanza ambiciosa y responsiva de las ciencias es un proceso activo, intencional, altamente contextualizado y lleno de tensiones. Es una actividad que involucra la toma continua de decisiones con base en información recolectada en el salón de clases y los objetivos de aprendizaje. Las acciones que el maestro decida implementar deberán responder a las necesidades del momento, el contexto particular en el que trabaja, las características individuales de sus estudiantes y las restricciones impuestas por el currículo y el sistema escolar. Este tipo de enseñanza es una tarea compleja para la cual los docentes deben estar preparados. Para ello se requiere que los programas de formación sean congruentes con sus objetivos y enseñen a enseñar involucrando a los docentes en tareas que modelen la filosofía que se profesa. 


\section{Referencias}

Bennett, R. E. (201 1). Formative assessment: A critical review. Assessment in Education: Principles, Policy \& Practice, 18(1), 5-25.

Black, P. y Wiliam, D. (1998). Assessment and classroom learning. Assessment in Education, 5(1), 7-74.

Black, P. y Wiliam, D. (2009). Developing the theory of formative assessment. Educational Assessment, Evaluation and Accountability, 21, 5-31.

Chi, M. T. H. y Wylie, R. (2014). The ICAP framework: Linking cognitive engagement to active learning outcomes. Educational Psychologist, 49, 219-243.

Coffey, J. E.; Hammer, D.; Levin, D. M. and Grant, T. (2011). The missing disciplinary substance of formative assessment. Journal of Research in Science Teaching, 48(10), 1109-1136.

Cowie, B. y Bell, B. (1999). A model of formative assessment in science education. Assessment in Education: Principles, Policy \& Practice, 6(1), 101-116.

Darling-Hammond, L. y Bransford, J. (eds.) (2005). Preparing teachers for a changing world. John Wiley \& Sons: San Francisco, CA.

Doyle, W. y Carter, K. (1984). Academic tasks in classrooms. Curriculum Inquiry, 14, 129-149.

Fang, Z. (1996). A review of research on teacher beliefs and practices. Educational Research, 38(1), 47-65.

García, J. J. y Rentería, E. (2011). Modelización de problemas para desarrollar habilidades de experimentación. Tecné, Episteme y Didaxis (TED), 29, 44-64.

Gess-Newsome, J. (1999). Secondary teachers' knowledge and beliefs about subject matter and its impact on instruction. En J. Gess-Newsome and N. G. Lederman, (eds.), Examining pedagogical content knowledge: The construct and its implications for science education (pp. 51 -94). Kluwer Academic Publishers: Dordrecht, The Netherlands.

Kang, H.; Windschitl, M.; Stroupe, D. y Thompson, J. (2016). Designing, launching, and implementing high quality learning opportunities for students that advance scientific thinking. Journal of Research in Science Teaching. doi:10.1002/ tea. 21329

Levin, D. M. y Richards, J. (2011). Learning to attend to the substance of student thinking in science. Science Educator, 20(2), $1-11$.

Mercer, N. (2000). Words and minds: How we use language to think together, Londres: Routledge.

Mora, W. M. y Parga, D. L. (2008). El conocimiento didáctico del contenido en química: integración de las tramas de contenido histórico-epistemológicas con 
las tramas de contexto-aprendizaje. Tecné, Episteme y Didaxis (TED), 24, 56-81.

National Research Council (NRC). (2011). A framework for K-12 science education: Practices, crosscutting concepts, and core ideas. Committee on a Conceptual Framework for New K-1 2 Science Education Standards. The National Academies Press: Washington, DC.

National Research Council (NRC). (2013). The next generation science standards. The $\mathrm{Na}$ tional Academies Press: Washington, DC.

National Science Foundation. (2016). Tools for Ambitious Science Teaching (TAST). Recuperado de http://ambitiousscienceteaching.org/.

Organisation for Economic Cooperation and Development (OECD). (2009). Creating effective teaching and learning environments: First results from Talis. Teaching and Learning International Survey, OECD.

Osborne, J. F. and Dillon J. (2008). Science education in Europe, London: Nuffield Foundation.

Porlan, R. y del Pozo, R. M. (2004). The conceptions of in-service and prospective primary school teachers about the teaching and learning of science. Journal of Science Teacher Education, 15(1), 39-62.

Robertson, A. M.; Scherr, R. and Hammer, D. (eds.) (2016). Responsive teaching in science and mathematics. Taylor \& Francis: Nueva York.

Roberson, B. and Franchini, B. (2014). Effective task design for the TBL classroom. Journal on Excellence in College Teaching, 25(3\&4), 275-302.

Roehrig, G. H. and Kruse, R. A. (2005). The role of teachers' beliefs and knowledge in the adoption of a reform-based curriculum. School Science and Mathematics, 105(8), 412-422.
Russ, R.; Coffey, J.; Hammer, D. and Hutchison, P. (2009). Making classroom assessment more accountable to scientific reasoning: A case for attending to mechanistic thinking. Science Education, 93(5), 875-891.

Shepard, L. (2005). Linking formative assessment to scaffolding. Educational Leadership, 63(3), 66-70.

Talanquer, V. (2013). School chemistry: the need for transgression, Science \& Education, 22, 1757-1773.

Talanquer, V.; Bolger, M. y Tomanek, D. (2015). Exploring prospective teachers' assessment practices: Noticing and interpreting student understanding in the assessment of written work. Journal of Research in Science Teaching, 52(5), 585-609.

Talanquer, V. y Pollard J. (2010). Let's teach how we think instead of what we know. Chemistry Education Research \& Practice, 11, 74-83. [Visite: https://sites.google.com/site/chemicalthinking/ para obtener más detalles sobre el currículo y https://sites.google.com/site/ ctinteractives/ para explorar simuladores interactivos asociados].

Thompson, I. (2014). Designing tasks in secondary education: Enhancing subject understanding and student engagement. Routledge: Nueva York, NY.

Van de Pol, J.; Volman, M. and Beishuizen, J. (2010). Scaffolding in teacher-student interaction: A decade of research. Educational Psychology Review, 22, 271-296.

Vilches, A. y Gil, D. (2007). La necesaria renovación de la formación del profesorado para una educación científica de calidad. Tecné, Episteme y Didaxis (TED), 22, 67-85.

Windschitl, M.; Thompson, J.; Braaten, M. and Stroupe, D. (2012). Proposing a core set 
of instructional practices and tools for teachers of science. Science Education, 96(5), 878-903.

\section{Para citar este artículo}

Vicente, T. (2017). Tres elementos fundamentales en la formación de docentes de ciencias. Revista de la Facultad de Ciencia y Tecnología-Tecné, Episteme y Didaxis (TED) 41 , 183-196. 